\title{
Anterior impingement syndrome in dancers
}

\author{
John William O'Kane · Nancy Kadel
}

Published online: 6 November 2007

(C) Humana Press 2007

\begin{abstract}
Anterior impingement is a common problem in dancers occurring primarily secondary to the repetitive forced ankle dorsiflexion inherent in ballet. Symptoms generally occur progressively and may respond to conservative treatment including addressing biomechanical faults that contribute to the problem. As impingement progresses, movements essential to ballet may become impossible and arthroscopic ankle surgery is often effective for both diagnosis and treatment, allowing athletes to return to dance.
\end{abstract}

Keywords Ballet - Dance $\cdot$ Ankle Impingement . Arthroscopy

\section{Introduction}

Injuries in classical ballet are common and often challenging to treat for a number of reasons. Many practitioners do not understand the physical demands of the sport or the terminology describing the common

J. W. O'Kane · N. Kadel

Department of Orthopaedics and Sports Medicine, University of Washington, Seattle, WA, USA

J. W. O'Kane

Department of Family Medicine, University of Washington, Seattle, WA, USA

J. W. O'Kane

Head Team Physician, University of Washington, Seattle, WA, USA

J. W. O'Kane ( $\square)$

U.W. Sports Medicine Clinic, Box 354060, Seattle, WA 981954060, USA

e-mail: jokane@u.washington.edu mechanisms of over use. Classical ballet dancers subject themselves to repetitive loads that require progressive training over hundreds of hours both increasing the risk of overuse injury and complicating rehabilitation that involves any rest from dance. The ability to dance en pointe (on the tips of the toes) for instance, requires progressive development of the kinetic chain from the back to the toes, any disruption of which may result in overuse injury anywhere in the chain.

Foot and ankle injuries that are more common in classical ballet are both anterior and posterior ankle impingement, flexor hallucis longus tendonitis, and stress fractures at the base of the second metatarsal and fibula [1]. This article will address the diagnosis and treatment of anterior ankle impingement including when performers should return to dance.

\section{Terminology}

Successful treatment of injuries in classical ballet starts with an understanding of the basic positions and common movements which can lead to overuse injury. The five basic positions involve maximum turnout of the hips to achieve the foot position. Inadequate hip turnout results in excessive knee or ankle external rotation and rolling in of the ankle to achieve the desired foot position (Fig. 1). Rolling in excessively stretches the medial ankle and compresses the anterior lateral ankle which can contribute to anterior impingement. Plié (Fig. 2) is the common dance movement contributing to anterior impingement and the "rolled in" position of the foot exaggerates the lateral compressive forces worsening the problem. 


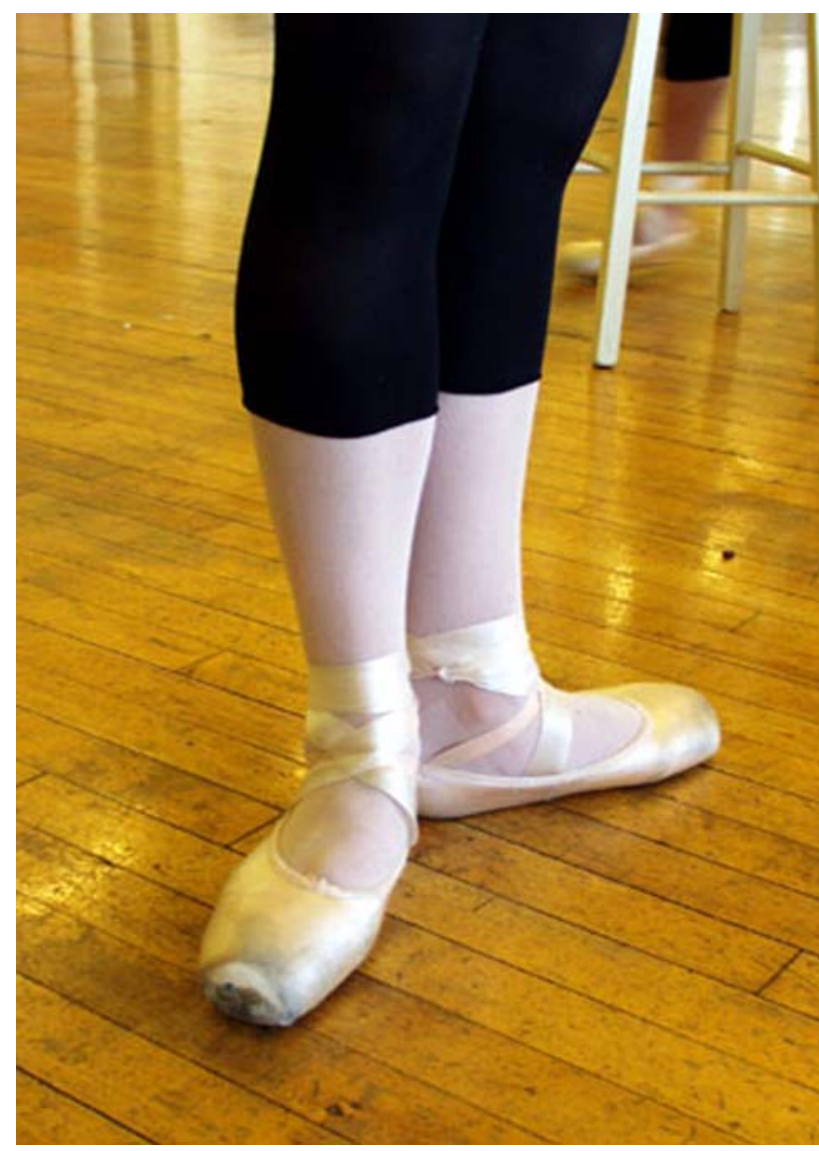

Fig. 1 Right foot demonstrates excessive pronation of ankle in attempt to exaggerate turnout

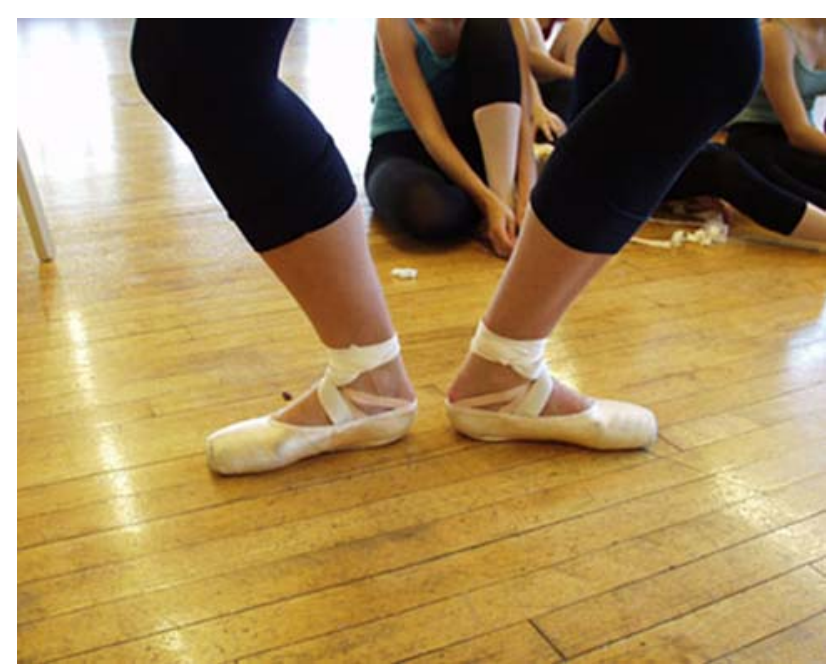

Fig. 2 Plié resulting in compressive force to anterior ankle

\section{Pathophysiology}

Anterior impingement in athletes is either secondary to hypertrophied soft tissue interposed in the anterior ankle joint or proliferation of osteophytes (Fig. 3) that limit the

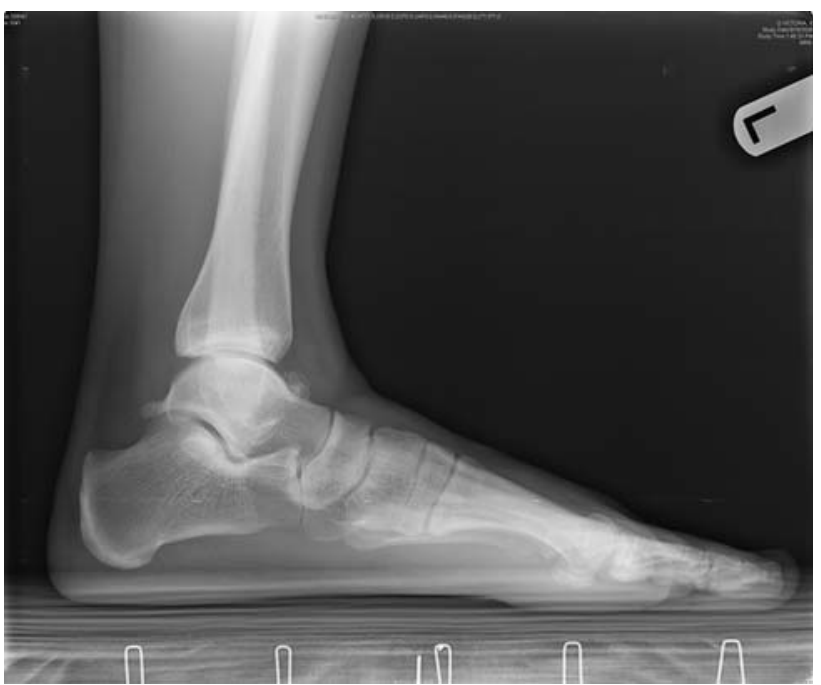

Fig. 3 Lateral ankle X-ray showing large talar osteophyte in patient with symptomatic anterior bony impingement

open space between the anterior lip of the tibia and the dorsal talar neck. Different theories have been proposed to account for the pathologic changes. Because impingement often occurs in athletes subject to forced plantar flexion (soccer players kicking or dancers en pointe (Fig. 4)) one proposed mechanism is that traction on the anterior capsule results in spurs that then impinge. Different studies have rebuked this theory. Tol et al. in a study of soccer players

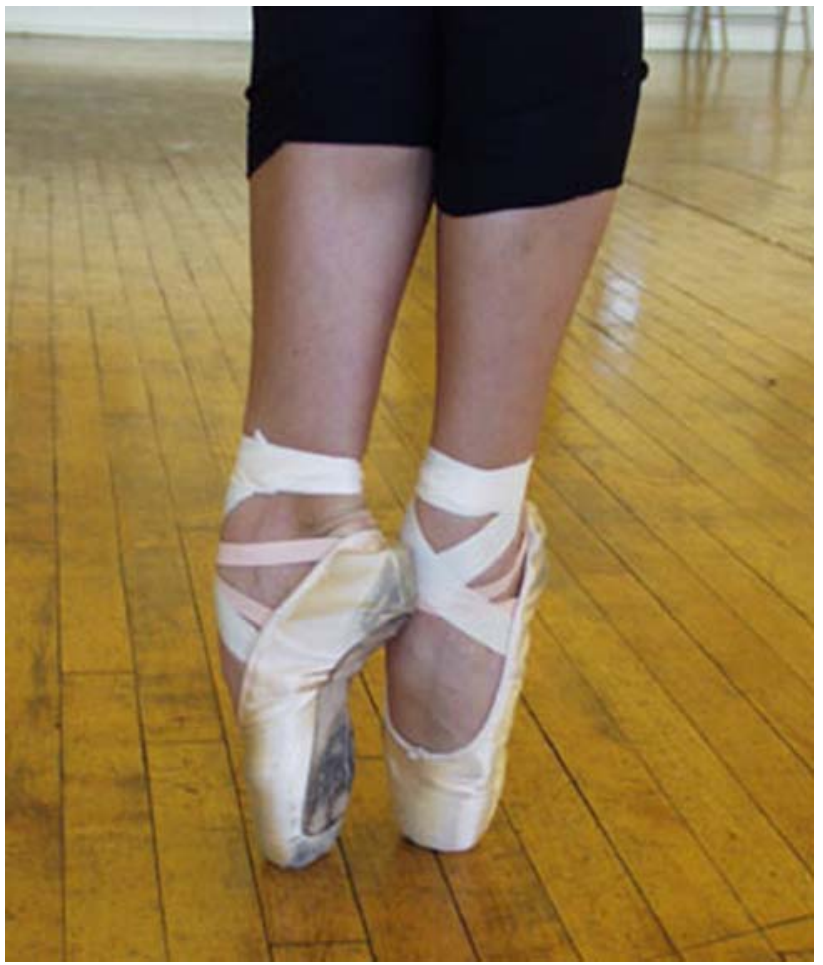

Fig. 4 Releve position 
found that the spurs typically occur in the location of ball impact without excessive plantar flexion being achieved suggesting that the impact and not traction of the capsule results in the spurring [2]. Tol in a cadaveric study demonstrated that the location of the osteophytes does not correspond with the attachment points of the capsule. He also located hypertrophied soft tissue in the anterior joint overlying the capsule that was compressed between the tibia and the talus with ankle dorsiflexion [3]. His conclusion was that traction could not be responsible for the spurs and that hypertrophied soft tissue in the anterior ankle was likely responsible for impingement symptoms.

Current thinking is therefore that osteophyte formation is secondary to repetitive ankle sprains or repetitive microtrauma as one would find with a ball impact or contact between the anterior tibia and talar neck with repetitive loaded dorsiflexion in ballet. Once the osteophytes form, the space available for ankle dorsiflexion diminishes likely resulting in soft tissue impingement, inflammation, hypertrophy, and pain [4]. The presence of osteophytes does not always correlate with impingement suggesting that soft tissue changes must also be necessary. This is supported by the finding that anterior osteophytes are very common in dancers occurring in 59\% without symptoms of impingement. This study also demonstrated that talar osteophytes were particularly prominent in dancers [5]. It is also well accepted that soft tissue impingement can occur without the presence of osteophytes. Ankle arthroscopy has demonstrated anterior impingement from the anterior inferior tibiofibular ligament [6] and from hypertrophied synovium in the absence of significant osteophytosis [7].

\section{Diagnosis}

Athletes with anterior impingement often have a history of multiple ankle sprains and present complaining of pain and some swelling in the anterior ankle aggravated by loaded dorsiflexion of the ankle. Experienced dancers may not have a history of ankle sprain but complain of increasing pain through training and performance especially with plié positions and landing from jumps. Dancers are often very sensitive to body position and note a loss of dorsiflexion in the affected ankle usually noted as a loss of ability to plié on the affected side (Fig. 5). The key physical finding is tenderness to palpation along the anterior joint line. Osteophytes at the anterior lateral or anterior medial joint line can often be palpated with the foot in some plantar flexion. Weightbearing full dorsiflexion of the ankle with the foot flat will often show some limitation on the affected side and reproduce symptoms.

Nihall et al. in a review of 11 dancers with anterior ankle impingement treated arthroscopically provides a profile of

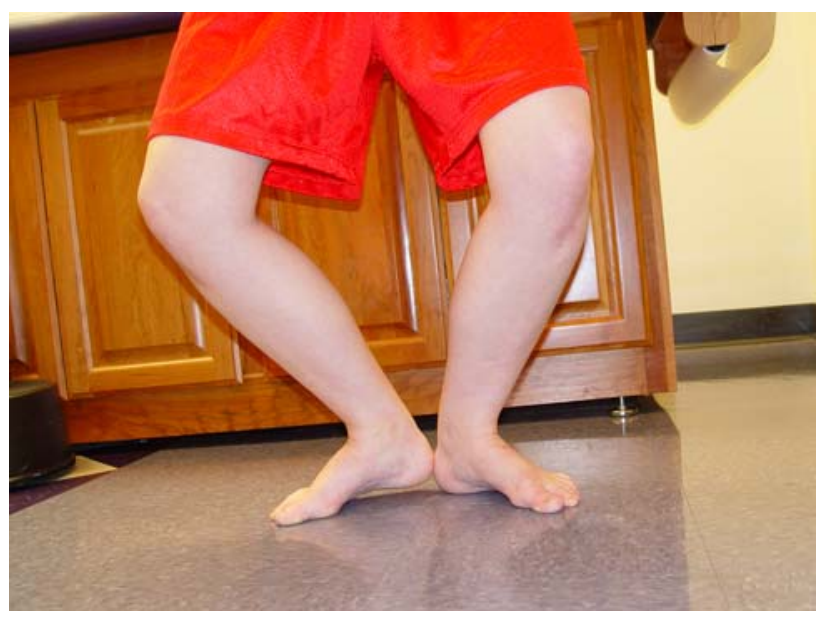

Fig. 5 Dancer with right ankle impingement limiting plié. She is compensating by lifting heel

the typical dancer presenting with impingement [7]. The patients included 7 women and 4 men all professional or pre-professional dancers performing primarily ballet. The women had been dancing for a mean of 18 years and the men 10 years. They danced between 12 and 30 h/week. All reported prior ankle trauma, primary sprains and two fractures. All had tenderness inferiorly or anterior-laterally and all had pain with plié.

Milloy et al. in 2003 describe a sign they found useful to diagnose synovial soft tissue impingement in the anterior lateral ankle in patients complaining of persistent pain following inversion injury [8]. They first manually dorsiflex the symptomatic ankle and note pain that reproduced symptoms. They repeat the maneuver (for left ankle) with right palm on calcaneus and thumb applying pressure to the lateral gutter. Increased pain with this maneuver is theoretically secondary to the displacement of hypertrophic synovium in to the joint and denotes a positive test. Seventy-three patients underwent ankle arthroscopy. Forty-one had a positive test and 37 of those had synovial hypertrophy in the latter gutter (sensitivity of $94.8 \%$ ). Of 32 patients with negative sign, 2 had synovial hypertrophy (specificity of $88 \%$ ).

Imaging of suspected anterior impingement starts with plain weightbearing radiographs of the ankle. As discussed above, the diagnosis is primarily clinical but imaging is necessary to rule out other causes of anterior ankle pain and confirm the presence and location of osteophytes that need to be removed to achieve the best surgical results. Plain radiographs including AP and mortise views are less helpful for the diagnosis of impingement, but will demonstrate other causes of ankle pain including fracture, mortise disruption, OCD, and arthritis. The identification of arthritis is important to plan treatment because the surgical results with arthroscopy are significantly worse if arthritis 
is present [9]. A lateral view will often demonstrate the tibial and talar osteophytes associated with anterior impingement. Adding an oblique view with the beam $45^{\circ}$ craniocaudal and the foot plantar flexed with the leg $30^{\circ}$ externally rotated will increase the detection of medially located tibial and talar osteophytes [10[k1]]. CT scanning can also be used to identify osteophytes contributing to impingement and in areas with easy access to CT, this is often the preferred method of identifying bony changes in the ankle, especially if plain films are non-diagnostic in the setting of clinical impingement.

It is well accepted that anterior impingement can occur without the formation of osteophytes secondary to entrapment of hypertrophied synovium or torn anterior lateral ligaments [11[k2]]. MRI has been studied to evaluate its accuracy to identify purely soft tissue impingement. T1 and proton density axial views demonstrating thickened synovium in the anterior lateral joint space or hypertrophy of the anterior talo-fibular ligament are the findings most consistently associated with impingement at arthroscopy [12[k3]]. The identification of chondral injury to the anterior aspect of the joint is also helpful as this finding decreases the likelihood that arthroscopic debridement of anterior osteophytes and hypertrophied tissue will result in significant improvement.

\section{Treatment}

\section{Conservative}

Relative activity limitation such as avoiding jumps, avoiding forcing of demi-plié, combined with attention to correct technique to reduce pronation may improve the symptoms of anterior impingement in dancers.

Physical therapy with focus on the entire kinetic chain, joint mobilization, and taping may resolve symptoms in some dancers.

Use of a night splint or removable walking cast brace can be tried briefly, and a single intra-articular corticosteroid injection may be used in select cases when other conservative measures prove ineffective.

\section{Operative}

Ankle arthroscopy is a useful tool both for diagnosis and treatment of persistent ankle pain following injury and it may be the only way to definitely diagnose and treat soft tissue impingement from an abnormal fibrous band [13[k4]]. Arthroscopy also provides highly effective treatment of bony and anterior soft tissue ankle impingement provided there is no significant associated chondral lesion in the anterior ankle [9].

Surgical treatment is reserved for those dancers who have failed conservative measures. If initial radiographic studies fail to show osseous impingement, then an ankle arthroscopy is performed. Thickened synovial tissue, and or the thickened distal fascicle of the anteroinferior tibiofibular ligament is debrided with a shaver. Any articular cartilage damage is addressed with debridement, drilling, and/or microfracture.

For those dancers with osseous impingement, either talar neck or tibial, arthroscopic or open ostectomy can be performed. Any hypertrophied tissue is debrided along with the osteophytes. Open treatment is not recommended when an osteochondral defect is present, but for talar neck or distal tibial osteophytes without other intra-articular pathology, a small anteromedial arthrotomy can be used effectively.

Post-operative care includes use of an ankle cryo-cuff to control edema and pain, alternating with a removable boot for ambulation. Patients are allowed to bear weight as pain allows unless an osteochondral defect has been drilled. In that case, patients are maintained strictly non-weightbearing for 6 weeks. After 10-14 days (for those with simple debridement or ostectomy), the patient is allowed to wean out of the boot. Physical therapy is begun to gradually increase range of motion and strength, with attention to proprioception exercises. Dancers may start Pilates, floor barre, and return to class gradually as comfort allows. Dancers are counseled that return to full performance may be limited for up to 6 months.

\section{References}

1. Khan K, Brown J, Way S, et al. Overuse injuries in classical ballet. Sports Med 1995;19(5):341-57.

2. Tol JL, Slim E, van Soest AJ, van Dijk CN. The relationship of the kicking action in soccer and anterior ankle impingement syndrome. A biomechanical analysis. Am J Sports Med 2002;30(1):45-50.

3. Tol JL, van Dijk CN. Etiology of the anterior ankle impingement syndrome: a descriptive anatomical study. Foot Ankle Int 2004;25(6):382-6.

4. van Dijk CN. Anterior and posterior ankle impingement. Foot Ankle Clin 2006;11(3):663-83.

5. Stoller SM, Hekmat F, Kleiger B. A comparative study of the frequency of anterior impingement exostoses of the ankle in dancers and nondancers. Foot Ankle 1984;4(4):201-3.

6. Bassett FH III, Gates HS III, Billys JB, Morris HB, Nikolaou PK. Talar impingement by the anteroinferior tibiofibular ligament. A cause of chronic pain in the ankle after inversion sprain. J Bone Joint Surg Am 1990;72(1):55-9.

7. Nihal A, Rose DJ, Trepman E. Arthroscopic treatment of anterior ankle impingement syndrome in dancers. Foot Ankle Int 2005;26(11):908-12. 
8. Molloy S, Solan MC, Bendall SP. Synovial impingement in the ankle. A new physical sign. J Bone Joint Surg Br 2003;85(3): 330-3.

9. Urguden M, Soyuncu Y, Ozdemir H, Sekban H, Akyildiz FF, Aydin AT. Arthroscopic treatment of anterolateral soft tissue impingement of the ankle: evaluation of factors affecting outcome. Arthroscopy 2005;21(3):317-22.

10. Tol JL, Verhagen RA, Krips R, et al. The anterior ankle impingement syndrome: diagnostic value of oblique radiographs. Foot Ankle Int 2004;25(2):63-8.
11. Ferkel RD, Karzel RP, Del Pizzo W, Friedman MJ, Fischer SP. Arthroscopic treatment of anterolateral impingement of the ankle. Am J Sports Med 1991;19(5):440-6.

12. Duncan D, Mologne T, Hildebrand H, Stanley M, Schreckengaust $\mathrm{R}$, Sitler $\mathrm{D}$. The usefulness of magnetic resonance imaging in the diagnosis of anterolateral impingement of the ankle. J Foot Ankle Surg 2006;45(5):304-7.

13. Takao M, Uchio Y, Naito K, Fukazawa I, Ochi M. Arthroscopic assessment for intra-articular disorders in residual ankle disability after sprain. Am J Sports Med 2005;33(5):686-92. 